\title{
ARTICLE \\ Role of infection control in combating antibiotic resistance
}

\author{
A C Whitelaw, MB BCh, MSc, FCPath (SA) (Micro) \\ Division of Medical Microbiology, Department of Pathology, Faculty of Medicine and Health Sciences, Stellenbosch University and \\ National Health Laboratory Service, Tygerberg Hospital, Cape Town, South Africa
}

\section{Corresponding author: A C Whitelaw (awhitelaw@sun.ac.za)}

\begin{abstract}
Infection control has been identified as one of the key interventions in controlling the threat of antibiotic resistance. Reducing the transmission of multidrug-resistant organisms (MDROs) reduces the need for broad-spectrum antibiotics in particular, while interventions that decrease the risk of infection have an impact on the use of any antibiotic. Hand hygiene remains the cornerstone of decreasing the transmission of MDROs. Alcohol-based hand rubs are a cheap, effective and convenient means of performing hand hygiene. Patients colonised or infected with MDROs should be placed on contact precautions, although implementation remains challenging in resourcelimited environments. Screening for certain MDROs may play a role in curbing transmission of these organisms. If implemented, screening must be part of a comprehensive infection control strategy. In resource-limited settings, the costs and potential benefits of screening programmes need to be carefully weighed up. Care bundles have been shown to reduce the incidence of common healthcare-associated infections, including catheter-associated urinary tract infection, ventilator-associated pneumonia, central line-associated bloodstream infection and surgical site infection. These bundles are relatively inexpensive, and can play an important role in reducing antibiotic use and improving clinical outcomes.
\end{abstract}

S Afr Med J 2015;105(5):421. DOI:10.7196/SAMJ.9650

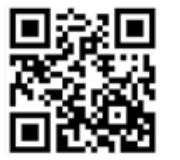

Antimicrobial resistance (AMR) is now well recognised as a global health threat ${ }^{[1]}$ that impacts on human health and may potentially have a major effect on the global economy. ${ }^{[2]}$ The United States Centers for Disease Control and Prevention (CDC) has identified four core actions to combat this challenge, i.e. surveillance, antibiotic stewardship, improved drugs and diagnostics, and preventing spread. ${ }^{[3]}$ While infection control is a very broad topic, this article focuses on measures to prevent the spread of multidrug-resistant organisms (MDROs) in healthcare settings and interventions to prevent infection.

\section{Transmission of multidrug-resistant organisms}

Transmission of any infectious agent (whether multidrug resistant or not) implies the presence of a source of the organism, a susceptible host, and a transfer mechanism. Most infectious agents in the healthcare setting are transmitted from humans (most commonly patients, but also healthcare workers (HCWs) or visitors), although transmission from environmental sources has also been well described. ${ }^{[4,5]}$ The greater the number of patients colonised with a particular MDRO (the 'colonisation pressure'), the greater the chance of transmission. ${ }^{[5]}$ Colonised HCWs have also been implicated in the transmission of infectious agents, ${ }^{[6]}$ but are thought to be a far less common source. ${ }^{[5]}$

Although organisms can be transmitted to any patient in hospital, certain patients are at higher risk of colonisation (and therefore of potential infection), including those with underlying medical conditions, those with compromised defences (such as indwelling devices, burns), and those who have undergone recent surgery.

There are three broad mechanisms by which organisms can be transmitted in healthcare settings: ${ }^{[4]}$ contact, droplet transmission and airborne transmission. Droplet transmission refers to organisms spread by large respiratory droplets (e.g. pertussis, diphtheria, certain respiratory viruses), while airborne transmission applies to organisms spread on droplet nuclei that remain suspended for prolonged periods (e.g. Mycobacterium tuberculosis, measles, varicella). Neither of these are a major route of transmission for MDROs, except for MDR tuberculosis, which is not the focus of this article.

Contact spread is the most important means of transmission for the vast majority of MDROs in healthcare settings, and can be either direct or indirect. Direct contact transmission occurs when an infected or colonised person comes into physical contact with a susceptible host. Indirect contact transmission implies the presence of some intermediate vehicle - either human or an inanimate object. Although transmission by items such as contaminated equipment, shared toys and poorly sterilised equipment is possible, the most important means of transmission is via the hands of HCWs.

\section{Prevention of transmission}

As the hands of HCWs are the most important means of transmission of MDROs, the importance of hand hygiene cannot be overstated. A number of studies have shown that improvements in hand hygiene are associated with lower healthcare-associated infection rates, and/or reductions in MDRO transmission and acquisition..$^{[7-10]}$ Nonetheless, compliance with hand hygiene recommendations is often poor, with studies reporting rates as low as $20-30 \% .{ }^{[11-13]}$

The adequate disinfection of hands is easily accomplished by using an alcoholic hand rub, with or without additional disinfectants, e.g. chlorhexidine. The alcohol is responsible for the immediate killing of transient flora on the hands, while the additional agents may exert a residual effect, limiting reacquisition of organisms for a limited period (although the evidence is mainly based on in vitro simulations, and the additional benefit of preventing transmission has not been studied well).

Alcohol hand rubs are superior to both ordinary soap and water and medicated soap and water with regard to the efficacy of removal of micro-organisms. This is based on numerous in vitro simulations and clinical evidence. ${ }^{[14]}$ As an example, a 6-year observation study at a tertiary centre in the USA compared 3 years of medicated soap use with 3 years of alcoholic hand rub use, and showed reductions in acquisition of both methicillin-resistant Staphylococcus aureus (MRSA) and vancomycin-resistant Enterococcus (VRE) (21\% and 


\section{Your 5 Moments for Hand Hygiene}
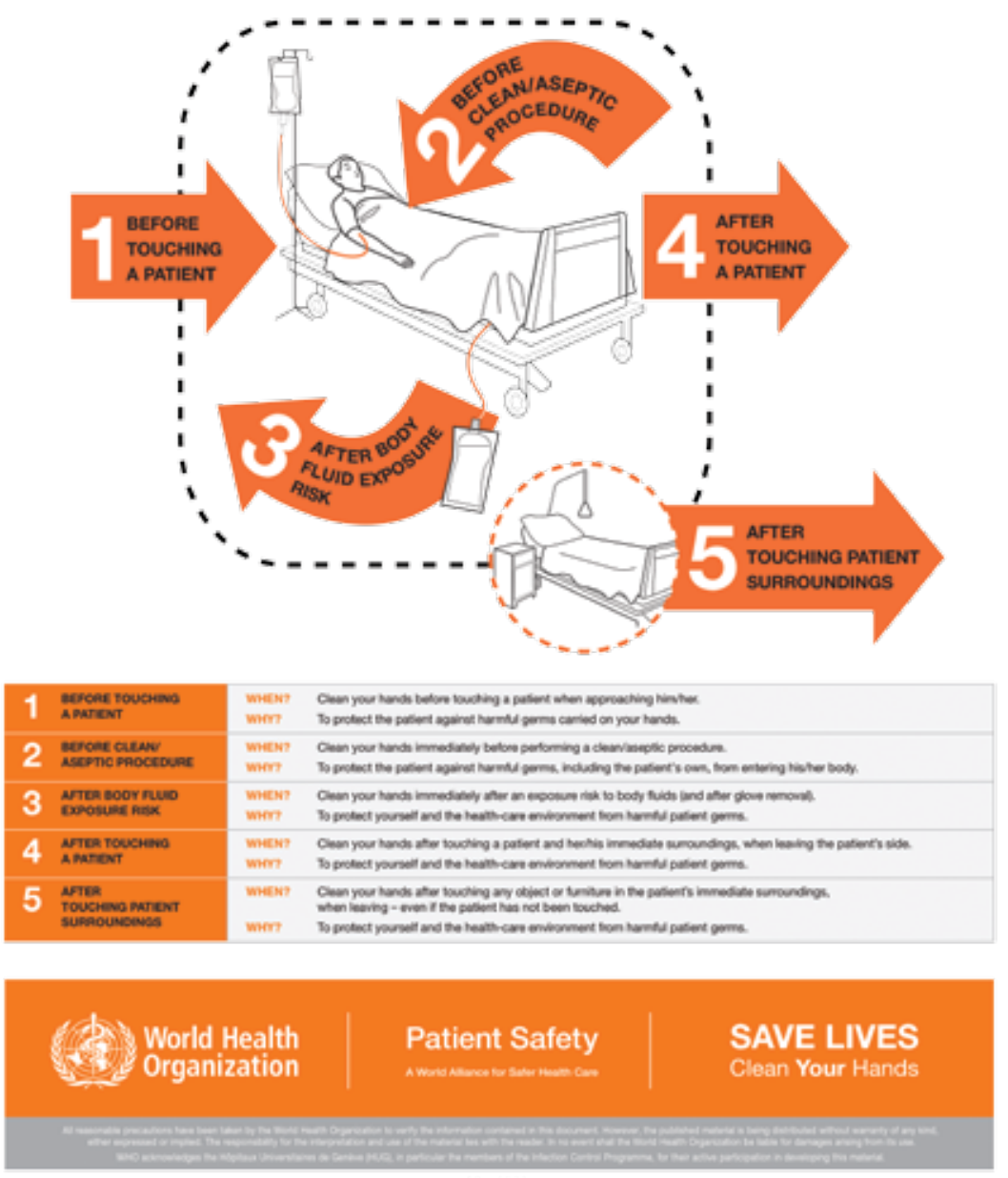

Fig. 1. The World Health Organization's 'Your 5 Moments for Hand Hygiene' campaign. ${ }^{[14,16]}$

$41 \%$ reductions, respectively). ${ }^{[15]}$ This may be due to the greater efficacy of alcohol and improved compliance with hand hygiene, with hand rubs being more easily accessible. Alcohol hand rubs can be manufactured very easily and cheaply if commercial products are not available. One suggested recipe is as follows: ${ }^{[14]}$

- ethanol $96 \% \mathrm{v} / \mathrm{v}, 833.3 \mathrm{~mL}$

- hydrogen peroxide $\left(\mathrm{H}_{2} \mathrm{O}_{2}\right) 3 \%, 41.7 \mathrm{~mL}$

- glycerol 98\%,14.5 mL.

Make up to $1000 \mathrm{~mL}$ with distilled water or boiled and cooled water and shake gently to mix the contents.

$\mathrm{H}_{2} \mathrm{O}_{2}$ is added to help eliminate contaminating spores in the bulk solutions, and glycerol acts as an emollient to improve acceptability. Neither of these items plays a role in the actual hand disinfection process.

Other important factors to remember with regard to the use of alcohol-based hand rubs are:

- If hands are visibly soiled, they should first be cleaned with soap and water - before using hand rubs.

- Alcohol is less effective at removing spores; hence, when caring for patients with suspected or confirmed Clostridium difficile infections, medicated soap is preferred.

The World Health Organization is promoting the 'Your 5 Moments for Hand Hygiene' campaign (Fig. 1), ${ }^{[1416]}$ which recommends that hand hygiene be practised as follows:

- before patient contact

- before a clean or aseptic procedure
- after contact with body fluids

- after patient contact

- after contact with the patient's environment.

\section{Measures beyond hand hygiene}

Other measures have been advocated to reduce the risk of transmission of MDROs. The $\mathrm{CDC}^{[4]}$ recommends that all patients colonised or infected with epidemiologically important pathogens (including MDROs) that are spread by contact be placed on contact precautions. This consists of isolating the patient in a room (or placing the patient with others with the same organism), use of gloves for all patient contact, and use of gowns/aprons during patient contact. Where isolation rooms are limited, patients with MDROs may need to be nursed in open wards. However, even there, every effort should be made to implement the glove and gown components of contact precautions. These recommendations are based on expert opinion and knowledge of the transmission mechanism, but there is limited evidence that contact precautions make a significant difference to transmission of MDROs, over and above good hand hygiene and adherence to basic infection control principles. ${ }^{[5,17-19]}$ Many studies include multiple interventions (improved staffing levels, education campaigns, hand hygiene campaigns) in addition to contact precautions, and it is difficult to identify which has the greatest impact.

There have been concerns about the impact of isolation on the mental wellbeing of patients. ${ }^{[20]}$ The additional burden of having patients on contact precautions may lead to a reduction in HCW compliance with these precautions. ${ }^{[21]}$ Even given these concerns, there is evidence that implementation of contact precautions does improve hand hygiene compliance, ${ }^{[19]}$ and it is still recommended for patients with MDROs. However, the importance of adequate staffing to sustain contact precautions and standard infection control practices cannot be overstated.

\section{Screening and}

\section{decolonisation}

There is good evidence that active screening of preoperative patients for MRSA, with decolonisation of carriers, results in reductions in postoperative sepsis caused by MRSA and other pathogens. ${ }^{[2]}$ The same effect has been described where all patients were decolonised with nasal mupirocin and chlorhexidine washes without the added cost of screening. ${ }^{[23]}$ Chlorhexidine washes alone have also been shown to reduce the 
acquisition of MDROs and development of healthcare-associated bacteraemia by $23 \%$ and $28 \%$, respectively. ${ }^{[24]}$ This raises the question of whether the application of intranasal mupirocin has benefit over chlorhexidine washes. The effect of chlorhexidine washes or wipes on infections with MDR Gram-negative bacteria specifically has not been as well described, and this, together with the descriptions of emerging chlorhexidine resistance, has led to some concerns about the implementation of routine chlorhexidine washes as a decolonisation strategy. ${ }^{[25]}$

Surveillance cultures for carbapenem-resistant Enterobacteriaceae (CRE) have been advocated in a number of reports and recommendations as part of an overall strategy to combat this specific MDRO. ${ }^{[26,27]}$ Screening has been a part of successful CRE control measures described in a variety of settings. ${ }^{[28,29]}$ However, as before, all these studies have included other interventions and it is very difficult to determine the effect of surveillance cultures alone on CRE control. There is evidence that CRE can be controlled without the use of surveillance cultures, ${ }^{[18]}$ and in resource-limited settings in particular it may be more appropriate to increase overall infection control capacity than to focus on screening.

Decolonisation strategies have not been well described for any of the resistant Gram-negative organisms. Some studies have demonstrated emergence of resistance during CRE decolonisation regimens (which usually include colistin), ${ }^{[30-32]}$ and this, allied to limited evidence of its efficacy, strongly suggests that decolonisation for multiresistant Gram-negative bacteria should not be attempted. If active screening for CRE carriage is carried out, the main intervention for carriers consists of isolation/cohorting and enhanced infection control measures. The duration of isolation for CRE (and for many other MDROs) is not well defined. CRE can be carried for $\geq 12$ months, ${ }^{[33]}$ and if patients are identified as CRE carriers, it may be valuable to be able to identify them on readmission to hospital. Screening to document elimination of carriage can be attempted; however, it has been suggested that there should be at least two negative cultures (and possibly a negative polymerase chain reaction test) before a known CRE carrier patient can be regarded as no longer carrying this organism. ${ }^{[34]}$ This is an area where more research is required.

\section{Prevention of infection}

While many infection control interventions focus on reducing the transmission of organisms, it is as important to identify measures to reduce the risk of infection. Fewer infections translate into reductions in antibiotic use and better patient outcomes. Many professional bodies and groups have adopted the use of 'care bundles', whereby different interventions focused on the same infection are combined and practised as a standard of care. In South Africa, this is being driven by the 'Best Care ... Always' (BCA) campaign (http://www. bestcare.org.za/home). The four infections addressed by the BCA bundles are catheter-associated urinary tract infection (CA-UTI), ventilator-associated pneumonia (VAP), central line-associated bloodstream infection (CLABSI), and surgical site infection (SSI).

Although there are some differences in the components of the bundles drawn up by different groups, and there is room for addition or modification, the components of the BCA bundles are as follows:

\section{CA-UTI}

- Avoid unnecessary catheterisation

- Insert catheters using an aseptic technique

- Maintain catheters based on recommended guidelines

- Review the need for catheterisation daily and remove the catheter as soon as possible.
VAP

- Elevation of the head of the bed to $45^{\circ}$ (or $30^{\circ}$ if $45^{\circ}$ not possible)

- Subglottic drainage of secretions

- Assess readiness for extubation daily

- Oral care and cleaning with chlorhexidine

- Initiation of enteral feeding within 24 - 48 hours of admission.

\section{CLABSI}

- Maximal barrier precautions when inserting line

- Chlorhexidine antisepsis when inserting line

- Selection of optimal catheter insertion suite (weighing infection and complication risks)

- Hand hygiene when caring for line

- Review the need for line daily, and remove as soon as possible.

SSI

- Appropriate antibiotic prophylaxis (timing, choice, duration)

- Appropriate hair removal (clippers or depilatory creams, not shaving)

- Postoperative glucose control

- Maintain postoperative normothermia.

Although some of the abovementioned components may be difficult to implement, the majority are relatively simple, require minimal resources, and when implemented can result in a dramatic reduction in infection. The incidence of SSI after colorectal surgery was $2 \%$ in patients who received all six components of a bundle, and $17 \%$ in those who received only one component. ${ }^{[35]}$ A multicentre before-andafter study in Korea showed a reduction in VAP rates from 4.08/1 000 ventilator-days to $1.16 / 1000$ ventilator-days after implementation of a VAP bundle. ${ }^{[36]}$ Reductions in VAP rates after implementing bundles have been associated with reduced ventilation-days and length of stay in the intensive care unit (ICU). ${ }^{[37]}$

Implementation of CLABSI bundles has resulted in reductions in CLABSI rates of up to $56 \%$; $^{[38]}$ despite different studies using different bundles, there is a consistent reduction in the rates of these infections. ${ }^{[38-40]}$ Introduction of bundles for CLABSI, VAP and CA-UTI in ICUs of a Saudi Arabian hospital resulted in reductions in the rates of all three infections ( $86 \%$ for VAP, $63 \%$ for CLABSI and $50 \%$ for CA-UTI) ${ }^{[41]}$

\section{Conclusion}

Infection control has a major role to play in combating the threat of AMR, and many interventions that have proven benefit are relatively inexpensive. Chief among these is hand hygiene. The challenge remains the appropriate implementation of these interventions, and sustaining them once immediate threats are over. Infection control should no longer be seen as the sole responsibility of the infection control practitioner, but that of every HCW.

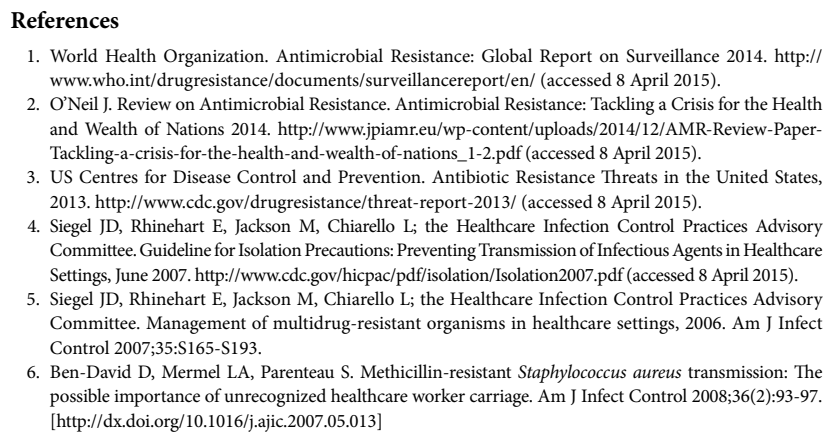

6. Ben-David D, Mermel LA, Parenteau S. Methicillin-resistant Staphylococcus aureus transmission: The possible importance of unrecognized healthcare worker carriage. Am J Infect Control 2008;36(2):93-97. [http://dx.doi.org/10.1016/j.ajic.2007.05.013] 
7. Kaier K, Hagist C, Frank U, Conrad A, Meyer E. Two time-series analyses of the impact of antibiotic consumption and alcohol-based hand disinfection on the incidences of nosocomial methicillinresistant Staphylococcus aureus infection and Clostridium difficile infection. Infect Control Hosp Epidemiol 2009;30(4):346-353. [http://dx.doi.org/10.1086/596605]

8. Stone SP, Fuller C, Savage J, et al. Evaluation of the national Cleanyourhands campaign to reduce Staphylococcus aureus bacteraemia and Clostridium difficile infection in hospitals in England and Wales by improved hand hygiene: Four year, prospective, ecological, interrupted time series study. BMJ 2012;3(344):e3005. [http://dx.doi.org/10.1136/bmj.e3005]

9. Helder OK, Brug J, van Goudoever JB, Looman CW, Reiss IK, Kornelisse RF. Sequential hand hygiene promotion contributes to a reduced nosocomial bloodstream infection rate among very low-birth weight infants: An interrupted time series over a 10-year period. Am J Infect Control 2014;42(7):718722. [http://dx.doi.org/10.1016/j.ajic.2014.04.005]

10. De Angelis G, Cataldo MA, De Waure C, et al. Infection control and prevention measures to reduce the spread of vancomycin-resistant enterococci in hospitalized patients: A systematic review and meta-
analysis. J Antimicrob Chemother 2014;69(5):1185-1192. [http://dx.doi.org/10.1093/jac/dkt525]

11. Marra AR, Edmond MB. New technologies to monitor healthcare worker hand hygiene. Clin Microbiol Infect 2014;20(1):29-33. [http://dx.doi.org/10.1111/1469-0691.12458]

12. Tenna A, Stenehjem EA, Margoles L, Kacha E, Blumberg HM, Kempker RR. Infection control knowledge, attitudes, and practices among healthcare workers in Addis Ababa, Ethiopia. Infect Control Hosp Epidemiol 2013;34(12):1289-1296. [http://dx.doi.org/10.1086/673979]

13. Block L, Habicht R, Oluyadi FO, et al. Variability in hand hygiene practices among internal medicine interns. Am J Infect Control 2013;41(11):1107-1108. [http://dx.doi.org/10.1016/j.ajic.2013.03.303]

14. World Health Organization (WHO). WHO guidelines on hand hygiene in healthcare 2009. http:// whqlibdoc.who.int/publications/2009/9789241597906_eng.pdf (accessed 8 April 2015).

15. Gordin FM, Schultz ME, Huber RA, Gill JA. Reduction in nosocomial transmission of drug resistant bacteria after introduction of an alcohol-based handrub. Infect Control Hosp Epidemiol 2005;26(7):650-653.

16. Sax H, Allegranzi B, Uçkay I, Larson E, Boyce J, Pittet D. 'My five moments for hand hygiene’' A usercentred design approach to understand, train, monitor and report hand hygiene. Journal of Hospital Infection 2007;67:9-21.

17. Huskins WC, Huckabee CM, O'Grady NP, et al. Intervention to reduce transmission of resistant bacteria in intensive care. N Engl J Med 2011;364:1407-1418. [http://dx.doi.org/10.1056/NEJMoa 1000373]

18. Kim NH, Han WD, Song KH, et al. Successful containment of carbapenem-resistant Enterobacteriacea by strict contact precautions without active surveillance. American Journal of Infection Control by strict contact precautions without active surveillance. American
2014;42(12):1270-1273. [http://dx.doi.org/10.1016/j.ajic.2014.09.004]

19. Harris AD, Pineles L, Belton B, et al. Universal glove and gown use and acquisition of antibioticresistant bacteria in the ICU: A randomized trial. J Am Med Assoc 2013;310(15):1571-1580.

20. Bearman G, Stevens MP. Control of drug-resistant pathogens in endemic settings: Contact precautions, controversies, and a proposal for a less restrictive alternative. Curr Infect Dis Rep 2012;14(6):620-626. [http://dx.doi.org/10.1007/s11908-012-0299-8]

21. Dhar S, Marchaim D, Tansek R, et al. Contact precautions: More is not necessarily better. Infect Control Hosp Epidemiol 2014;35(3):213-221. [http://dx.doi.org/10.1086/675294]

22. Van Rijen M, Bonten M, Wenzel R, Kluytmans J. Mupirocin ointment for preventing Staphylococcus aureus infections in nasal carriers. Cochrane Database Syst Rev 2008;8(4):CD006216. [http://dx.doi. org/10.1002/14651858.CD006216.pub2]

23. Huang SS, Septimus E, Kleinman K, et al. Targeted versus universal decolonization to prevent ICU infection. N Engl J Med 2013;368(24):2255-2265. [http://dx.doi.org/ 10.1056/NEJMoa1207290]

24. Climo MW, Yokoe DS, Warren DK, et al. Effect of daily chlorhexidine bathing on hospital-acquired infection. N Engl J Med 2013;368(6):533-542. [http://dx.doi.org/ 10.1056/NEJMoa1113849]
25. Tacconelli E, Cataldo MA, Dancer SI, et al. ESCMID guidelines for the management of the infection control measures to reduce transmission of multidrug-resistant Gram-negative bacteria in hospitalized control measues to re tansmission of multing

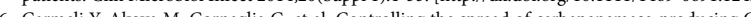

6. Carmeli Y, Akova M, Cornaglia G, et al. Controlling the spread of carbapenemase-producing Gramnegatives: Therapeutic approach and infection control. Clin Microbiol Infect 2010;16(2):102-111

27. Centers for Disease Control and Prevention (CDC). Guidance for control of infections with carbapenemresistant or carbapenemase-producing Enterobacteriaceae in acute care facilities. MMWR Morb Morta Wkly Rep 2009;58(10):256-260.

28. Poulou A, Voulgari E, Vrioni G, et al. Imported Klebsiella pneumoniae carbapenemase-producing K. pneumoniae clones in a Greek hospital: Impact of infection control measures for restraining the dissemination. J Clin Microbiol 2012;50(8):2618-2623. [http://dx.doi.org/10.1128/JCM.00459-12]

29. Schwaber MJ, Carmeli Y. An ongoing national intervention to contain the spread of carbapenemresistant Enterobacteriaceae. Clin Infect Dis 2014;58(5):697-703. [http://dx.doi.org/10.1093/cid/cit795]

30. Oren I, Sprecher H, Finkelstein R, et al. Eradication of carbapenem-resistant Enterobacteriaceae gastrointestin colonization with non-abstale on antibiotic treatment: A prospective controlled trial. Am Jnfect Control 2013;41(12):1167-1172. [http://dx.doi.org/10.1016/.jajic.2013.04.018]

31. Lubbert C. Faucheux S, Becker-Rux D, et al. Rapid emergence of secondary resistance to gentamicin and colistin following selective digestive decontamination in patients with KPC-2-producing Klebsiella pneumoniae: A single-centre experience. International Journal of Antimicrobial Agents 2013;42(6):565-570. [http://dx.doi.org/ 10.1016/j.ijantimicag.2013.08.008]

32. Brink AJ, Coetzee J, Corcoran C, et al. Emergence of OXA-48 and OXA-181 carbapenemases among Enterobacteriaceae in South Africa and evidence of in vivo selection of colistin resistance as consequence of selective decontamination of the gastrointestinal tract. J Clin Microbiol 2013;51(1):369372. [http://dx.doi.org/10.1128//CM.02234-12]

33. Zimmerman FS, Assous MV, Bdolah-Abram T, Lachish T, Yinnon AM, Wiener-Well Y. Duration of carriage of carbapenem-resistant Enterobacteriaceae following hospital discharge. Am J Infect Contro 2013;41(3):190-194. [http://dx.doi.org/10.1016/j.ajic.2012.09.020]

34. Feldman N, Adler A, Molshatzki N, et al. Gastrointestinal colonization by KPC-producing Klebsiella pneumoniae following hospital discharge: Duration of carriage and risk factors for persistent carriage. Clinical Microbiology and Infection 2013;19(4):E190-E196. [http://dx.doi.org/10.1111/1469-0691.12099]

35. Waits SA, Fritze D, Banerjee M, et al. Developing an argument for bundled interventions to reduce surgical site infection in colorectal surgery. Surgery 2014;155(4):602-606. [http://dx.doi.org/100.1016/j. 13.12 .004

36. Eom IS, Lee MS, Chun HK, et al. The impact of a ventilator bundle on preventing ventilator-associated pneumonia A multicenter study. Am J Infect Control 2014;42(1):34-37. [http://dx.doi.org/10.1016/j.ajic.2013.06.023]

37. Rello J, Afonso E, Lisboa T, et al. A care bundle approach for prevention of ventilator-associated pneumonia. Clin Microbiol Infect 2013;19(4):363-369. [http://dx.doi.org/10.1111/j.1469-0691.2012.03808.x]

38. Jeffries HE, Mason W, Brewer M, et al. Prevention of central venous catheter-associated bloodstrean infections in pediatric intensive care units: A performance improvement collaborative. Infect Control Hosp Epidemiol 2009;30(7):645-651. [http://dx.doi.org/10.1086/598341]

39. Miller MR, Niedner MF, Huskins WC, et al. Reducing PICU central line-associated bloodstream infection 3-year results. Pediatrics 2011;128(5):e1077-e1083. [http://dx.doi.org/10.1542/peds.2010-3675]

40. Schulman J, Stricof R, Stevens TP, et al. Statewide NICU central-line-associated bloodstream infection rates decline after bundles and checklists. Pediatrics 2011;127(3):436-444. [http://dx.doi.org/10.1542/ peds.2010-2873]

41. Al-Tawfiq JA, Amalraj A, Memish ZA. Reduction and surveillance of device-associated infections in adult intensive care units at a Saudi Arabian hospital, 2004-2011. Int J Infect Dis 2013:17(12): 1207-e1211. [http://dx.doi.org/10.1016/j.ijid.2013.06.015] 